Results No difference were noted in: BMI and in duration of SLE in groups investigated. The borderline difference was existed between $\mathrm{N}$ and OPO group in duration of CS treatment $(p=0.049)$. The relation between particular data where statistical significance was obtained in groups investigated is presented in the Table 1.

\begin{tabular}{llll}
\multicolumn{4}{l}{ Abstract FRI0147 Table 1} \\
\hline & N:OPE & N:OPO & OPE:OPO \\
\hline Age at onset of SLE & ns & $* * *$ & $* * *$ \\
Age at study & ns & $* * *$ & $* * *$ \\
BMD & $* * *$ & $* * *$ & ns \\
\hline$* * * p<0.0001$. & &
\end{tabular}

Conclusion Low body mass density was found in elder group of patients with SLE, where the disease started later in perimenopause. Duration of disease, and duration of corticosteroide treatment did not influence BMD. Further studies will consider other factors possibly significant in development of osteoporosis in SLE (subsets of disease, immunological disturbances, cumulative dose of CS and known risk factors for osteoporosis.

\section{FRI0148 2-YEARS BONE MINERAL DENSITY FOLLOW-UP IN PATIENTS WITH SYSTEMIC LUPUS ERYTHEMATOSUS}

'J Rosa, ${ }^{2} V$ Tesa, ${ }^{1} \mathrm{C}$ Dostál, ${ }^{1} \mathrm{~J}$ Zadina, ${ }^{1} \mathrm{H}$ Hulejová, ${ }^{1} \mathrm{~J}$ Kafková, ${ }^{3} \mathrm{I}$ Malá, ${ }^{1} \mathrm{E}$ Rogie. ${ }^{1}$ Institute of Rheumatology; ${ }^{2}$. Clinic of Internal Medicine, General University Hospital; ${ }^{3}$ University of Economics, Prague, Czech Republic

\subsection{6/annrheumdis-2001.183}

Background Systemic lupus erythematosus (SLE) and its treatment comprises several potential risk factors of osteoporosis.

Objectives We evaluated bone mineral density (BMD) in a cohort of ambulatory patients with SLE and determined the influence of corticosteroids and disease-related variables at the baseline and during 24-months follow-up.

Methods BMD of the lumbar spine, proximal femur, and total body was measured by dual energy X-ray absorptiometry (DEXA) in 61 SLE patients (52 premenopausal women, 9 men; mean age 32,3 and 36 years, respectively) and 61 healthy controls matched for age and sex. Several disease-related variables including treatment characteristics were recorded. 50 patients (40 women, 9 men) and 37 patients ( 31 women, 6 men) were followed for another 12 and 24 months, respectively.

Results With few exceptions the BMD values of all sites were significantly reduced in both women and men ( $\mathrm{p}$ values $<0,001-$ 0,05 ) as compared to controls. During 12 months of follow-up a significant BMD decrease was seen in neck (both women and men), total body (women) and in proximal femur (men). In women current corticosteroid dose adversely influenced total body mineral content development. Continuous BMD loss has been detected during another 12 months in a subgroup of patients taking $>10 \mathrm{mg}$ prednisone daily. Generally, there was no further BMD decline in the second year of study, presumably due to lower average daily prednisone dose compared to that at the start of study (10,0 vs. $14,3 \mathrm{mg}$ in women, 11,3 vs. 22,4 in men).

Conclusion BMD in premenopausal women and in men with SLE is significantly reduced at all measured sites and is adversely influenced by duration of the illness (proximal femur) and prednison cumulative dose (lumbar spine, total body). This decline occurs presumably early during the corticosteroid treatment and further bone loss is limited to patients taking $>10 \mathrm{mg}$ prednisone daily.

This work has been supported by IGA MZ ÈR 5035-3.

\section{OP0100 INFECTIOUS COMPLICATIONS IN SYSTEMIC LUPUS ERYTHEMATOSUS}

JJ Rovensky, F Mateicka. Department of Immunopharmacology, Research Institute of Rheumatic Diseases, Piestany, Slovakia

10.1136/annrheumdis-2001.184

Background In the last decades long time survival of patients with systemic diseases of connective tissue improved dramatically, not only due to new therapeutic methods but also thanks to prompt diagnostics of infections and their effective treatment.

In spite of this, infection complications constitute a considerable determinant of mortality in patients with systemic lupus erythematosus (SLE). In these patients, morbidity by bacterial, viral and fungal diseases is higher compared to patients suffering from other rheumatic diseases. There are many reasons why it is so, the most important include variability of infection agents virulence factors, immunopathogenic features of diseases (dysbalance of T-lymphocytes populations, cytokine dysbalance), activity of disease, factors related to it and also the therapy (corticosteroids and immunosuppressants).

Objectives Authors present detailed results of longitudinal retrospective analysis of morbidity and mortality caused by infections summarised from studies published up to now in the world literature as well as clinical analysis of infectious complications in a group of SLE patients treated in Research Institute of Rheumatic Diseases.

Methods A group of 135 patients with SLE diagnosed according ARA criteria was studied for infections induced by community acquired pathogens and opportune microorganisms. Clinical and laboratory predictors of infection development in these patients were assessed as well.

Results Actual morbidity for infection was 36,3\% and mortality in connexion to the infection was $1,5 \%$. From the community acquired pathogens the most frequent were infections caused by enterobacteria and Staphylococcus aureus. Fungal infections caused by Candida spp. were predominant in the group of opportune infections. Regarding anatomical site of infection the most frequent were infections of lower respiratory tract and urogenital system. Significant correlation between past infection, activity of the disease (evaluated by ECLAM and SLEDAI indices), proteinuria, leucopenia and high corticoids dosage were revealed. In multiple regression model only activity of the disease as a risk factor for development of infection in patients with SLE was designated.

Conclusion For effective control and prevention of infectious complications in systemic diseases of connective tissues, changing pathogenicity time trends and resistance to anti-infectious therapy (antibiotics, antifungal and antiviral agents) have to be considered. 